\title{
The use of fondaparinux for the treatment of venous thromboembolism in a patient with heparin-induced thombocytopenia and thrombosis caused by heparin flushes
}

\author{
Alex C Spyropoulos' \\ Sharyl Magnuson' \\ Sei Keng Koh² \\ 'Clinical Thrombosis Center, Lovelace \\ Medical Center, Albuquerque, NM, \\ USA; '2Department of Pharmacy, \\ Singapore General Hospital, Singapore
}

Correspondence: Alex C Spyropoulos Clinical Thrombosis Center, Lovelace Medical Center, 500 Walter St, Ste 30I, Albuquerque, NM 87102, USA

$\mathrm{Tel}+\mathrm{I} 5052627874$

Fax + I 5052627040

Email alex.spyropoulos@abqhp.com

\begin{abstract}
Heparin-induced thrombocytopenia (HIT) is an immunologic drug reaction characterized by paradoxical association with venous and arterial thrombosis. The syndrome is caused by IgG antibodies that are reactive against complexes of platelet factor 4 and heparin. Fondparinux does not bind to platelet factor 4, is structurally too short to induce an antibody response, and could in theory be a useful agent to treat HIT. A 69-year-old white female presented with a lower extremity extensive iliofemoral deep vein thrombosis after a right total knee arthroplasty and was subsequently found to have a pulmonary embolism. The patient was noted to have heparin flushes during her operation. Her platelet drop decreased $>50 \%$ from baseline during initiation of antithrombotic therapy. She was started on subcutaneous fondaparinux $7.5 \mathrm{mg}$ once daily injection. Her serotonin release assay and enzyme-linked immunosorbent assay for heparin antibodies were positive for HIT. Her platelet count nadir was $60 \times 10^{3} / \mathrm{mm}^{3}$ on day 5 and the platelet count rebounded after 8 days of fondaparinux therapy. No recurrent thrombotic or bleeding events were noted throughout her therapy. Anecdotal reports have shown that fondaparinux can be a useful agent to treat HIT with or without thrombosis.
\end{abstract}

Keywords: fondaparinux, heparin-induced thrombocytopenia with thrombosis (HITT)

Fondaparinux (GlaxoSmithKline, Middlesex, UK) is a new synthetic pentasaccaride that binds to antithrombin and potentiates antithrombin inhibition of factor Xa. It does not bind to platelet factor 4 and is unlikely to cause immunoallergic heparin-induced thrombocytopenia (HIT) (Dager et al 2004). Data had shown that fondaparinux is structurally too short to induce an antibody response and could be a useful agent to treat HIT (Efird et al 2006).

\section{Case report}

A 69-year-old white female presented to the Clinical Thrombosis Center on March 5th, 2007, with a lower extremity extensive iliofemoral deep vein thrombosis (DVT) confirmed by Doppler ultrasonography, after a right total knee arthroplasty on February 19th, 2007. The patient had received aspirin prophylaxis $325 \mathrm{mg}$ twice daily during the postoperative period. She was started on subcutaneous enoxaparin injection (sanofiaventis, Paris, France) at $75 \mathrm{mg}$ twice daily and warfarin $7.5 \mathrm{mg}$ once daily by the emergency department on March 4th, 2007.

Her medical history included hypertension, degenerative joint disease, and dyslipidemia. Her past surgical histories included 3 cesarean sections, hysterectomy, and parathyroidectomy. Her family history was negative for venous thromboembolic 
disease. Her medications included verapamil, triamterene/ hydrochlorothiazide, lovastatin, aspirin, naproxen sodium, acetaminophen, vitamin $\mathrm{C}$, vitamin $\mathrm{E}$, and a multivitamin. The patient denied any shortness of breath, pleurisy, hemoptysis, or low grade fevers or chills, but complained of dyspnea on exertion. Her blood pressure was 143/76 mm $\mathrm{Hg}$, temperature $97.2^{\circ} \mathrm{F}$, pulse 96 , and oxygen saturation $97 \%$. Her laboratories results were significant for white count $9.2 \times 10^{3} / \mathrm{mm}^{3}$, hematocrit $37.7 \%$, platelet count $81 \times 10^{3} / \mathrm{mm}^{3}$. Her prothrombin time $(\mathrm{PT})$, activated partial thromboplastin time (aPTT), and liver function tests were all within normal limits. Her serum creatinine was $0.8 \mathrm{mg} / \mathrm{dl}$. On physical examination, the right lower extremity showed some severe swelling, especially of the knee. Her lungs were clear to auscultation and cardiovascular examination showed regular rate and rhythm.

Her last platelet count was done preoperatively on February 9th, 2007 and revealed a normal platelet count of $293 \times$ $10^{3} / \mathrm{mm}^{3}$. In view of her thrombocytopenia, the patient was admitted to the hospital for closer observation. Her enoxaparin injection was discontinued and fondaparinux injection was started subcutaneously at a dose of $7.5 \mathrm{mg}$ daily as a bridge to dose-adjusted warfarin, which was continued at $5.0 \mathrm{mg}$ daily. She was also worked up for HIT and disseminated intravascular coagulation (DIC).

The patient came into the clinic following her hospitalization for her DVT and thrombocytopenia on March 9th, 2007. The patient complained of pain in her knees, increased cough and continued dyspnea on exertion. She had no complaints of pleurisy, hemoptysis, fevers or chills. In view of her continued dyspnea on exertion, a ventilation/perfusion scan was ordered. The leg swelling on her right was better and she reported less pain on the leg, and the patient was advised to place compression stockings on the affected extremity. There was no evidence of limb-gangrene or warfarin-induced skin necrosis.

Her fingerstick international normalized ratio (INR) was 5.1 and she was on subcutaneous fondaparinux injection $7.5 \mathrm{mg}$ daily ( 5 doses given to date) and warfarin $5 \mathrm{mg}$ daily. She was also on acetaminophen $\left(\right.$ Tylenol $^{\circledR}$ ) $500 \mathrm{mg}$ every 4 hours for her leg pain. Her platelet count was $60 \times 10^{3} / \mathrm{mm}^{3}$ and she tested positive for heparin-induced antiplatelet antibodies. The patient refused to be re-admitted to the hospital for treatment of suspected HIT-associated thrombosis (HITT) with parenteral direct thrombin inhibitors, and after careful discussion with the patient and her family, she agreed to continue treatment on an outpatient basis under careful supervision with subcutaneous fondaparinux. The patient was informed to hold her warfarin and continue fondaparinux until the next clinic visit. She had a repeat INR and complete blood count check on March 12th, 2007. The patient was also advised to stop her Tylenol.

Her platelet count was $100 \times 10^{3}$, fingerstick INR 3.6, hematocrit $38.8 \%$ at the next visit. She restarted warfarin at $2.5 \mathrm{mg}$ daily. The serotonin release assay (SRA) and repeat enzyme-linked immunosorbent assay (ELISA) HIT studies were still pending at the point of consultation. The right leg swelling and inner thigh calf erythema were slightly decreased. The patient was advised to place compression stockings. We were informed by the patient's daughter that the patient had received heparin flushes during the time of her operation.

The patient had her follow up 4 days later. Her V/Q scan showed a high probability for pulmonary embolism. The platelet count was $123 \times 10^{3} / \mathrm{mm}^{3}$, hematocrit $34.4 \%$. HITT was confirmed by high pretest probability (significant thrombocytopenia of $>50 \%$ from baseline associated with unfractionated heparin (UFH) and low molecular weight heparin (LMWH) exposure at most 25 days from baseline associated with venous thrombosis) and positive ELISA HIT results and SRA done on March 9th, 2007 (86\%). Her fingerstick INR was 4.1. Her warfarin dose was adjusted and fondaparinux was discontinued. She was counseled against any future use of heparins or LMWHs in view of her history of HITT. Her platelet counts are shown in Table 1.

\section{Discussion}

HIT is an immunologic drug reaction characterized by paradoxical association with venous and arterial thrombosis (Warkentin et al 1995; Warkentin and Kelton 2001). The venous thromboembolic events dominate over arterial thromboembolic events at a ratio of approximately 4:1 (Warkentin and Kelton 1996; Wallis et al 1999; Kelton 2005). The syndrome is caused by IgG antibodies that are reactive against complexes of platelet factor 4 and heparin (Amiral et al 1992).

HIT occurs 5-10 days post exposure to heparin. Thrombocytopenia can occur quickly, even minutes to hours in patient who have HIT antibodies post previous exposure to heparin (Warkentin 1999). HIT antibody formation is accompanied by an unexplained platelet count fall (usually $\geq 50 \%$ fall, even if the platelet count nadir remains $>150 \times 10^{9} / \mathrm{L}$ ), or by skin lesions at heparin injection sites or acute systemic reactions (eg, chills, cardiorespiratory distress) after intravenous heparin bolus administration (Warkentin and Greinacher 2004).The incidence of HIT 
Table I Platelet counts and clinical course during the observed period

\begin{tabular}{|c|c|c|}
\hline Date & Platelet count $\left(\times 10^{3}\right) / \mathrm{mm}^{3}$ & Remarks \\
\hline 2/9/2007 & 293 & Baseline platelet count. \\
\hline $2 / 19 / 2007$ & & Right total knee arthroplasty with aspirin $325 \mathrm{mg}$ BID given for thromboprophylaxis. \\
\hline $3 / 4 / 2007$ & & $\begin{array}{l}\text { Doppler CUS with extensive right lower extremity ileofemoral DVT. Patient treated with } \\
\text { enoxaparin } 75 \mathrm{mg} \text { SQ BID and warfarin } 7.5 \mathrm{mg} \text { daily. Patient complaining of dyspnea on exertion. }\end{array}$ \\
\hline $3 / 5 / 2007$ & 81 & $\begin{array}{l}\text { First visit to the Clinical Thrombosis Center and first platelet count done postoperation. } \\
\text { Enoxaparin discontinued, fondaparinux } 7.5 \mathrm{mg} \text { SQ daily started, warfarin continued, and patient } \\
\text { admitted to hospital. ELISA and SRA studies for HIT ordered. }\end{array}$ \\
\hline $3 / 6 / 2007$ & 93 & Platelet counts done while hospitalized. Fondaparinux and warfarin continued. \\
\hline $3 / 7 / 2007$ & 94 & $\begin{array}{l}\text { Platelet counts done while hospitalized. Patient discharged on fondaparinux } 7.5 \mathrm{mg} \text { SQ daily } \\
\text { and warfarin } 7.5 \mathrm{mg} \text { daily. }\end{array}$ \\
\hline $3 / 9 / 2007$ & 60 & $\begin{array}{l}\text { Platelet count done in the Clinical Thrombosis Center post-hospitalization. ELISA test positive } \\
\text { for heparin-induced antiplatelet antibodies. Patient refuses re-hospitalization. Warfarin } \\
\text { discontinued, fondaparinux } 7.5 \mathrm{mg} \text { continued.Ventilation-perfusion scan ordered. }\end{array}$ \\
\hline $3 / 12 / 2007$ & 100 & Warfarin restarted at $2.5 \mathrm{mg}$ daily, fondaparinux continued. \\
\hline $3 / 16 / 2007$ & 123 & $\begin{array}{l}\text { Repeat ELISA and SRA studies positive for HIT, Ventilation-perfusion scan positive for pulmonary } \\
\text { embolism, fondaparinux discontinued, fingerstick INR 4.I, warfarin dose adjusted. }\end{array}$ \\
\hline $3 / 20 / 2007$ & 179 & Patient doing well, no clinicalVTE recurrence, continue dose-adjusted warfarin, target INR 2.5. \\
\hline
\end{tabular}

Abbreviations: BID, twice daily; CUS, cranial ultrascopography; ELISA, enzyme-linked immunosorbent assay; INR, international normalized ratio; SQ, subcutaneous; SRA, serotonin release assay; VTE, venous thromboembolism.

varies with the patient type (Warkentin et al 2000) and the heparin preparation (Powers et al 1984). The frequency of HIT is greater in surgical than in medical patients. In orthopaedic patients given subcutaneous prophylactic heparin, the incidence is approximately $5 \%$ with UFH and $0.5 \%$ with LMWH (Warkentin et al 2000). HIT can occur in $0.5 \%$ to $1 \%$ of patients who receive lower doses of heparin (subcutaneous or flushes) or even the tiny amounts that leach from heparincoated catheters (Laster and Silver 1988; Kadidal et al 1999; Mayo et al 1999; Lawrence et al 2002). Only a minority of patients who form HIT antibodies during heparin therapy develop clinical HIT. Most patients who form HIT antibodies develop seroconversion without thrombocytopenia or thrombosis. The risk of HITT occurs predominantly in patients who also develop thrombocytopenia (Warkentin 1999).

The management of HIT includes the discontinuation of UFH and LMWH therapy, and the use of an alternative agent such as the heparinoids and the direct thrombin inhibitors. An alternative agent is required as the patient is still at substantial thrombosis (Kuo and Kovacs 2005) and in one retrospective analysis, $40 \%-61 \%$ of patients with HIT suffered from thrombosis after heparin cessation (Wallis et al 1999). Warfarin therapy is not recommended for the initial treatment of HIT until the platelet count is above $100 \times 10^{9} / \mathrm{L}$ as it may lead to warfarin-induced venous limb gangrene and skin necrosis (Warkentin et al 1997: Klein et al 2004; Srinivasan et al 2004).
The direct thrombin inhibitors lepirudin, argatroban, and bivalirudin have been used for the treatment of HIT (Efird and Kocker 2006). These agents have chemical structures, which are different from UFH and LMWH and do not generate or cross react with antiheparin-platelet factor 4 antibodies (or HIT antibodies) and are effective and safe for the prophylaxis or treatment of thrombosis (Hassell 2005). However, their use is complicated by the need for continuous intravenous infusion administration, monitoring using aPTT, dose modification based on hepatic or renal function, and acquisition cost. Hence, other HIT treatment options are essential (Efird and Kocker 2006).

Fondaparinux is a synthetic pentasaccharide that catalyzes the inhibition of factor Xa by antithrombin, resulting in the inhibition of thrombin generation (Kuo and Kovacs 2005). Data had shown that an increased risk for HIT-related antigen production is dependent on molecular weight and length of polysaccarides $(>2.4 \mathrm{kDa}$ and $>10$ saccharide units, respectively); and structural components for UFH and LMWH are above the minimum requirements for increasing the risk of these antigens (Table 2). Amiral and colleagues (1997) had found no cross reactivity of fondaparinux with antibodies to the heparin-PF4 complexes in his in vitro assay. Warkentin and colleagues (2005) found that anti-PF4/heparin antibodies were generated at similar frequencies in patients treated with fondaparinux or enoxaparin (Warkentin et al 2005). Although antibodies reacted equally well in vitro against 
Table 2 Comparison of factors associated with Increased HIT antigenicity (Efird and Kocker 2006)

\begin{tabular}{lll}
\hline Agent & $\begin{array}{l}\text { Average molecular } \\
\text { weight } \mathbf{( k D a )}\end{array}$ & Saccharide residues \\
\hline UFH & 15 & $\sim 45$ \\
Tinzaparin & $5.5-7.5$ & $\sim 15$ \\
Dalteparin & 5.0 & $\sim 15$ \\
Enoxaparin & 4.5 & $\sim 13$ \\
Fondaparinux & 1.7 & 5
\end{tabular}

Abbreviations: HIT, heparin-induced thrombocytopenia; UFH, unfractionated heparin/

PF4/UFH and PF4/LMWH, and sometimes weakly against $\mathrm{PF} 4 /$ danaparoid, none reacted against PF4/fondaparinux, including even those sera obtained from patients who formed antibodies during fondapaprinux treatment. Hence, despite similar immunogenicity of fondaparinux and LMWH, PF4/ fondaparinux, but not PF4/LMWH is recognized poorly by the antibodies generated, suggesting that the risk of HIT with fondaparinux, if likely, is very low. Recently, a single case report of HIT with the use of fondaparinux has been reported (Warkentin et al 2007).

Anecdotal reports have shown that fondaparinux could be a useful agent in the treatment of HIT (Lian et al 2003; Parody et al 2003; Bradner et al 2004; Herenberg et al 2004) and HITT (Kuo and Kovacs 2003; Rubin 2003).

Kuo and Kovacs (2003) administered fondaparinux to 5 surgical patients who developed heparin/PF4ELISA-confirmed HIT after receiving UFH for venous thromboprophylaxis. Thrombocytopenia occurred between 6 and 10 days after the initiation of heparin anticoagulation and the platelet count nadir ranged from $5 \times 10^{9} / \mathrm{L}$ to $79 \times 10^{9} / \mathrm{L}$. Pulmonary embolism $(n=2)$, deep vein thrombosis $(n=2)$, and left atrial and mechanical mitral valve thrombosis $(n=1)$ were noted. In all cases, UFH or LMWH was discontinued and patients were treated with subcutaneous injections of fondaparinux $7.5 \mathrm{mg}$ daily as soon as the clinical diagnosis of HIT was made and UFH therapy had stopped. All patients responded with the platelet count becoming normal between 2 and 9 days after the initiation of anticoagulation with fondaparinux. Warfarin was initiated (and fondaparinux continued) when the platelet count was $>100 \times 10^{9} / \mathrm{L}$. Fondaparinux was then discontinued when the target INR was $\geq 2.0$ for two consecutive days. No recurrence of thombosis occurred during the therapy.

Rubin (2003) reported a case of fondaparinux use in a 29-year-old pregnant female who had HIT during her first trimester of pregnancy secondary to UFH treatment for pulmonary embolism. She had a platelet count drop of more than $50 \%$ from baseline after 5 days of treatment with UFH.
The assay for HIT antibodies was positive. She was started on fondaparinux $2.5 \mathrm{mg}$ subcutaneously twice daily. No further complications occurred throughout her pregnancy and the baby was born healthy without any problems with delivery.

Our patient presented with a large ileofemoral DVT and thrombocytopenia after major orthopedic surgery in which she was thromboprophylaxed with a weak antithrombotic agent, aspirin. She was treated with fondaparinux for HITT that was verified by a high pretest probability as well as positive ELISA and SRA studies. Further evaluation revealed that the patient likely had a concurrent pulmonary embolism during her index event. Interestingly, her HITT could be due to the heparin flushes she had gotten during her surgery and aggravated by the LMWH (enoxaparin) that she received in the emergency department for initial treatment of her DVT, in which initial suspicion of HITT was missed. Our patient reported no complications or bleeding from the use of fondaparinux subcutaneous injections, and other than complete blood counts and INRs, no other routine monitoring was done, which enabled the patient to complete her course of treatment in the outpatient setting. Her platelets counts rebounded after 7 days of treatment and we are still following her progress. Her last known platelet count on March 20th, 2007 was $179 \times 10^{3}$. We had also used fondaparinux injection in 2 previous patients with suspected HIT with no further complications or bleeding, although their clinical course is less well described.

\section{Conclusion}

Heparin-induced thrombocytopenia (HIT) with or without thrombosis is an immunologic drug reaction caused by $\mathrm{IgG}$ antibodies that are reactive against complexes of platelet factor 4 and heparin. Antibodies against PF4/fondaparinux have been described during fondaparinux therapy and a single case report of HIT with the use of fondaparinux has been reported. However, unlike the approved agents that are used to treat HIT (argatroban, lepirudin, and bivalirudin), fondaparinux does not require routine laboratory monitoring. Anecdotal reports have shown that fondaparinux can be a useful agent to treat HIT or HITT, possibly in the outpatient setting, but further larger trials are needed to assess the above.

\section{References}

Amiral J, Bridey F, Dreyfus M, et al. 1992. Platelet factor 4 complexed to heparin is the target for antibodies generated in heparin-induced thrombocytopenia [Letter]. Thromb Haemost, 68:95-9.

Amiral J, Lormeau JC, Marfaing-Koka A, et al. 1997. Absence of crossreactivity of SR $90107 \mathrm{a} / \mathrm{ORG} 31540$ pentasaccharide with antibodies to heparin=PF4 complexes developed in heparin-induced thrombocytopenia. Blood Coagul Fibrinolysis, 8:114-17. 
Bradner J, Hallisey Rk, Duter DJ. 2004. Fondaparinux in the treatment of heparin-induced thrombocytopenia [abstract]. Blood, 104:1775.

Dager WE, Andersen J, Nutescu E. 2004. Special considerations with fondaparinux therapy: heparin induced thrombocytopenia and wound healing. Pharmacotherapy, 24(7 pt 2):88S-94S.

Efird LE, Kockler DR. 2006. Fondaparinux for thromboembolic treatment and prophylaxis of heparin-induced thrombocytopenia. Ann Pharmacother, 40:1383-7.

Harenberg J, Jorg I, Fenyvesi T. 2004. Treatment of heparin-induced thrombocytopenia with fondaparinux. Haematologica, 89:1017-18.

Hassell K. 2005. The management of patients with heparin-induced thrombocytopenia who require anticoagulation therapy. CHEST, 127:1S-8S.

Kadidal VV, Mayo DJ, Horne MK. 1999. Heparin-induced thrombocytopenia (HIT) due to heparin flushes: a report of three cases. $J$ Intern Med, 246:325-9.

Kelton JG. 2005. The pathophysiology of heparin-induced thrombocytopenia: biological basis for treatment. CHEST, 127:9S-20S.

Klein L, Galvez A, Klein O, et al. 2004. Warfarin-induced limb gangrene in the setting of lung adenocarcinoma. Am J Hematol, 76:176-9.

Kuo HM, Kovacs MJ. 2003. Successful treatment of heparin induced thrombocytopenia (HIT) with fondaparinux [abstract]. Blood, 102:1147.

Kuo KHM, Kovacs MJ. 2005. Fondaparinux: a potential new therapy for HIT. Hematology, 10:271-5.

Laster J, Silver D. 1988. Heparin-coated catheters and heparin-induced thrombocytopenia. $J$ Vasc Surg, 7:667-72.

Lawrence R, Attisha WK, Drexler A, et al. 2002. Delayed-onset heparininduced thrombocytopenia. Ann Intern Med, 136:210-15.

Lian EC, Chua L, Oberstein E. 2003. Long term use of fondaparinux in a patient with antiphospholidp syndrome, heparin-induced thrombocytopenia and refractoriness to coumarin [abstract]. Blood, 102:4206.

Mayo DJ, Cullinane AM, Merryman PK et al. 1999. Serologic evidence of heparin sensitization in cancer patients receiving heparin flushes of venous access devices. Support Care Cancer, 7:425-7.

Parody R, Oliver A, Souto JC, et al. 2003. Fondaparinux (Atrixa ${ }^{\circledR}$ ) as an alternative anti-thrombotic prophylaxis when there is hypersensitivity to low molecular weight and unfractionated heparins. Haematologica, 88:ECR32.
Powers PJ, Kelton JG, Carter CJ. 1984. Studies on the frequency of heparin-associated thrombocytopenia. Thromb Res, 33:439-43.

Rubin N. 2003. Treatment of heparin-induced thrombocytopenia with thrombosis (HITT) in pregnancy with fondaparinux [abstract]. Blood, 102:4190.

Srinivasan AF, Rice L, Bartholomew JR, et al. 2004. Warfarin-induced skin necrosis and venous limb gangrene in the setting of heparin-induced thrombocytopenia. Arch Intern Med, 164:66-70.

Wallis DE, Workman DL, Lewis BE, et al. 1999. Failure of early heparin cessation as treatment for heparin-induced thrombocytopenia. $\mathrm{Am} \mathrm{J}$ Med, 106:629-35.

Warkentin TE, Cook Rj, Marder VJ, et al. 2005. Anti-platelet factor 4/heparin antibodies in orthopedic surgery patients receiving antithormbotic prophylaxis with fondaparinux or enoxaparin. Blood, 106:3791-6.

Warkentin TE, Elavathi LJ, Hayward CP, et al. 1997. The pathogenesis of venous limb gangrene associated with heparin-induced thrombocytopenia. Ann Intern Med, 127:804-12.

Warkentin TE, Greinacher A. 2004. Heparin-induced thrombotopenia: recognition, treatment, and prevention. The Seventh ACCP Conference on Antithrombotic and Thrombolytic. CHEST, 126:311S-337S.

Warkentin TE, Kelton JG. 1996. A 14-year study of heparin-induced thrombocytopenia. Am J Med, 101:502-7.

Warkentin TE, Kelton JG. 2001. Delayed-onset heparin-induced thrombocytopenia and thrombosis. Ann Intern Med, 135:502-6.

Warkentin TE, Levine MN, Hirsh J, et al. 1995. Heparin-induced thrombocytopenia in patients treated with low molecular weight heparin or unfractionated heparin. $N$ Engl J Med, 332:1330-5.

Warkentin TE, Maurer BT, Aster RH. 2007. Heparin-induced thrombocytopenia associated with fondaparinux. $N$ Engl $J$ Med, 356:2653-5.

Warkentin TE, Sheppard JA, Horsewood P, et al. 2000. Impact of the patient population on the risk for heparin-induced thrombocytopenia. Blood, 96:1703-8.

Warkentin TE. 1999. Heparin-induced thrombocytopenia: a clinicopathologic syndrome. Thromb Haemost, 82:439-47. 
